\title{
Neutrino Experiments
}

\author{
R. D. McKeown \\ W. K. Kellogg Radiation Laboratory, California Institute of Technology, Pasadena, CA
}

\begin{abstract}
Recent studies of neutrino oscillations have established the existence of finite neutrino masses and mixing between generations of neutrinos [1]. The combined results from studies of atmospheric neutrinos, solar neutrinos, reactor antineutrinos and neutrinos produced at accelerators paint an intriguing picture that clearly requires modification of the standard model of particle physics. These results also provide clear motivation for future neutrino oscillation experiments as well as searches for direct neutrino mass and nuclear double-beta decay. I will discuss the program of new neutrino oscillation experiments aimed at completing our knowledge of the neutrino mixing matrix.
\end{abstract}

Keywords: Neutrino Mass; Neutrino Mixing; Neutrino Oscillations

PACS: $14.60 . \mathrm{Pq}, 26.65 .+\mathrm{t}, 28.50 . \mathrm{Hw}$

\section{HISTORICAL INTRODUCTION}

In 1930 Pauli boldly suggested the existence of the neutrino [2] to conserve energy and spin/statistics in nuclear beta decay. Several decades then passed before Reines and Cowan obtained experimental evidence [3] for the presence of antineutrinos emitted by nuclear reactors in the 1950's. During the following decade, Pontecorvo [4] developed the concept of neutrino oscillations, where the different flavors of neutrino could mix with potentially observable consequences if there were neutrino states with finite distinguishable masses.

Interest in neutrino masses and oscillations was increased by the mysterious deficit of solar neutrinos first reported by Davis in 1968 [5]. In addition, there was growing evidence that there was a significant amount of dark matter in the universe [6]. This prompted a variety of experimental studies focused on the discovery of neutrino oscillations. However, the first convincing evidence for neutrino oscillations was reported by the SuperKamiokande experiment in 1998 [7]. During the last 12 years, many other experiments have reported results establishing neutrino oscillations [1] and measuring many of the relevant parameters. Nevertheless, the picture is not complete and additional experiments are in progress and planned for the future.

\section{NEUTRINO OSCILLATIONS}

The phenomenon of neutrino oscillations occurs when the flavor eigenstates (i.e., states produced in weak interaction processes, $v_{e}, v_{\mu}$ and $v_{\tau}$ ) are not identical with the mass eigenstates. The neutrino flavor eigenstate produced in a weak process (such as nuclear beta decay) is then a superposition of the mass eigenstates, and the subsequent evolution of the state as it propagates through space involves slippage of the relative phases of the

CP1265, VIII Latin American Symposium on Nuclear Physics and Applications

edited by R. Alcaron, H. F. Arellano, P. L. Cole, and A. J. Kreiner

(C) 2010 American Institute of Physics 978-0-7354-0814-2/10/\$30.00 
mass eigenstates leading to flavor oscillations.

This is easily demonstrated in a 2 flavor approximation where there are 2 flavor eigenstates (e.g. $v_{e}$ and $v_{\mu}$ ) that are superpositions of 2 mass eigenstates $\left(v_{1}\right.$ and $v_{2}$, with masses $m_{1}$ and $m_{2}$ ). The mixing is described by a matrix involving one mixing angle $\theta$

$$
\left(\begin{array}{c}
v_{e} \\
v_{\mu}
\end{array}\right)=\left(\begin{array}{cc}
\cos \theta & \sin \theta \\
-\sin \theta & \cos \theta
\end{array}\right)\left(\begin{array}{l}
v_{1} \\
v_{2}
\end{array}\right)
$$

If a $v_{e}$ is produced in an experiment, it will develop a component of $v_{\mu}$ as it propagates through space. The probability of observing a $v_{\mu}$ oscillates in the propagation distance $L$, and the probability for observing the $v_{\mu}$ state is given by

$$
P\left(v_{e} \rightarrow v_{\mu}\right)=\sin ^{2}(2 \theta) \sin ^{2}\left[1.27 \Delta m^{2}\left(\mathrm{eV}^{2}\right) \frac{L(\mathrm{~m})}{E_{V}(\mathrm{MeV})}\right]
$$

where $\Delta m^{2} \equiv\left|m_{2}^{2}-m_{1}^{2}\right|$ is the difference in squared masses and $E_{V}$ is the neutrino energy.

The 3 flavor case is a straightforward generalization in which there are 3 mass eigenstates (with masses $m_{1}, m_{2}$, and $m_{3}$ ) and a $3 \times 3$ mixing matrix that depends on 3 mixing angles $\left(\theta_{12}, \theta_{23}\right.$, and $\left.\theta_{13}\right)$ plus a $C P$-violating phase $\delta$. The mixing matrix is denoted $U_{P M N S}$ ( for Pontecorvo [4], Maki, Nakagawa and Sakata [8]) and, for neutrino oscillation physics, can be conveniently written as a product of three matrices:

$$
U_{P M N S}=\left(\begin{array}{ccc}
1 & 0 & 0 \\
0 & c_{23} & s_{23} \\
0 & -s_{23} & c_{23}
\end{array}\right) \times\left(\begin{array}{ccc}
c_{13} & 0 & s_{13} e^{-i \delta} \\
0 & 1 & 0 \\
-s_{13} e^{i \delta_{C P}} & 0 & c_{13}
\end{array}\right) \times\left(\begin{array}{ccc}
c_{12} & s_{12} & 0 \\
-s_{12} & c_{12} & 0 \\
0 & 0 & 1
\end{array}\right)
$$

where $s_{i j} \equiv \sin \theta_{i j}$ and $c_{i j} \equiv \cos \theta_{i j}$. As discussed below, there are already significant experimental determinations of $\theta_{12}$ and $\theta_{23}$, so the matrix element in Eq. 3 involving the $C P$-violating phase $s_{13} e^{-i \delta}$ vanishes if $\theta_{13}=0$. Thus the angle $\theta_{13}$ can be viewed as the gateway to observation of $C P$ violation in the lepton sector.

The SuperKamiokande results are associated with the mixing between mass eigenstates 2 and 3 . In fact, the most significant determination of $\theta_{23}$ comes from measurements of the oscillations associated with atmospheric neutrinos by the SuperKamiokande collaboration [9]: $\sin ^{2} 2 \theta_{23}>0.92$ at $90 \%$ CL. The most precise value of $\Delta m_{23}^{2}$ is from the MINOS accelerator-based oscillation measurement [10]: $\Delta m_{23}^{2}=2.43 \pm 0.13 \times 10^{-3} \mathrm{eV}^{2}$.

Experimental information regarding the mixing angle $\theta_{12}$ and $\Delta m_{12}^{2}$ comes from combined fits of the KamLAND reactor neutrino oscillation measurement and solar neutrino data (assuming CPT invariance) (see below).

\section{REACTOR EXPERIMENTS}

Nuclear reactors are prolific sources of antineutrinos emitted by neutron-rich fission fragments. The flux and energy spectrum of the antineutrinos has been studied in great detail [11]. The energy spectrum of the $\bar{v}_{e}$ is a steeply falling spectrum (see Fig. 1) and 
depends slightly on the mix of fissionable material which varies during the fuel cycle of the reactor. Generally, if one has knowledge of the reactor power and fuel composition then one can predict the flux to 1-2\% accuracy.

The reactor antineutrino experiments utilize the inverse beta decay reaction on the proton

$$
\bar{v}_{e}+p \rightarrow e^{+}+n
$$

which enables determination of the antineutrino energy through energy conservation $E_{v}=E\left(e^{+}\right)+1.31 \mathrm{MeV}+\bar{E}_{n}$. (The average neutron recoil energy $\bar{E}_{n} \sim 10 \mathrm{keV}$ is a very small contribution.) The threshold for this reaction is $E_{v} \simeq 1.8 \mathrm{MeV}$, and the cross section increases roughly quadratically with $E_{v}$ above this value, as shown in Fig. 1 . The cross section for this process is strongly constrained by the neutron lifetime, and so is accurately known to better than $0.5 \%$ precision [12].

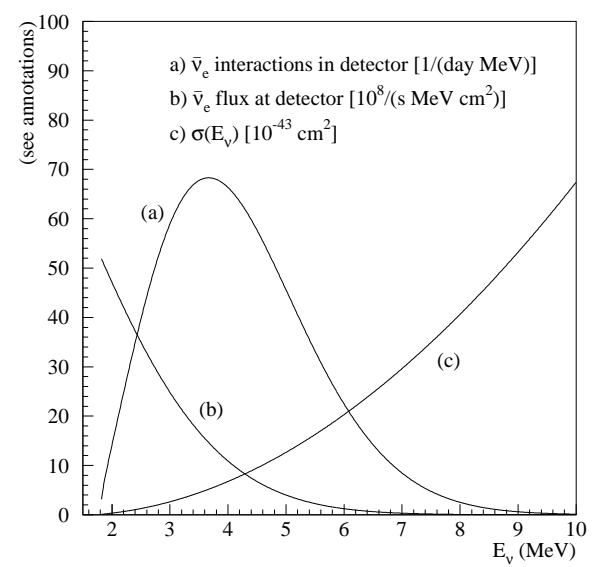

FIGURE 1. Reactor $\bar{v}_{e}$ spectrum, inverse beta decay cross section, and $\bar{v}_{e}$ interaction spectrum, from [11].

\section{CHOOZ and Palo Verde}

Following the Super-Kamiokande collaboration report of the observation of neutrino oscillations in atmospheric neutrinos two reactor neutrino experiments were built to further study this phenomenon and search for $\bar{v}_{e}$ disappearance. One was at the reactor plant in Chooz, France [13] with a baseline of $L \simeq 1 \mathrm{~km}$ and the other was at the Palo Verde plant in Arizona, USA [14] with a baseline of $L \simeq 0.8 \mathrm{~km}$. Both experiments detected the predicted flux of antineutrinos without oscillations, implying an upper limit on the value of $\sin ^{2} 2 \theta<0.13$ for $\Delta m^{2}=2.8 \times 10^{-3} \mathrm{eV}^{2}$ at $90 \%$ confidence. 


\section{KamLAND}

The 1000 Ton Kamioka Liquid scintillator AntiNeutrino Detector, KamLAND, was the first reactor neutrino experiment to observe neutrino oscillations. Use of the numerous nuclear power plants in Japan enabled the study of neutrino oscillations with typical baselines of $200 \mathrm{~km}$.

The KamLAND collaboration has reported [15] a significant $(>8 \sigma)$ deficit in the observed flux of antineutrinos relative to the no-oscillation hypothesis and obtained a measurement of the $\bar{v}_{e}$ energy spectrum showing a substantial distortion compared to the no-oscillation spectrum. When plotted as a function of the ratio $L_{0} / E_{v}$, with the reference baseline distance $L_{0} \equiv 180 \mathrm{~km}$, the spectrum shows an impressive oscillation pattern(see Fig. 2). Assuming CPT invariance, one can combine this information with the solar neutrino data to obtain

$$
\begin{aligned}
\Delta m_{12}^{2} & =7.59_{-0.21}^{+0.21} \times 10^{-5} \mathrm{eV}^{2} \\
\tan ^{2} \theta_{12} & =0.47_{-0.05}^{+0.06} .
\end{aligned}
$$

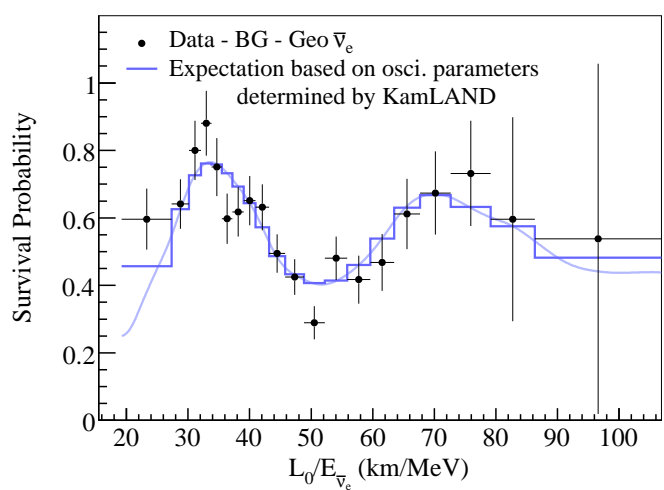

FIGURE 2. Distribution of observed events (with fitted geoneutrinos subtracted) in KamLAND vs. $L_{0} / E_{V}$ [15], where $L_{0} \equiv 180 \mathrm{~km}$. The histogram is the best fit to the neutrino oscillation hypothesis.

\section{Future Reactor Experiments}

We now have good knowledge of all the $\Delta m_{i j}^{2}$ and two of the mixing angles $\theta_{12}$ and $\theta_{23}$. However, we only have the upper limit for $\theta_{13}$ from $\mathrm{CHOOZ}$ and Palo Verde. As a result, there are new experiments being planned to determine this last mixing angle in the neutrino mixing matrix. As mentioned above, this parameter is also key for future attempts to study the $C P$-violating phase $\delta_{C P}$.

There are presently three new $\theta_{13}$ reactor antineutrino experiments being pursued. These all intend to utilize the method of comparing two identical detectors at different baselines to reduce systematic errors. The new experiments are Double-CHOOZ [16] located at the CHOOZ site, a new experiment in China, the Daya Bay experiment [17], 
and another called RENO in Korea [18]. A summary of the experimental parameters, schedules, and projected sensitivities is presented in Table 1.

TABLE 1. Parameters of the 3 new reactor experiments to study $\theta_{13}$.

\begin{tabular}{ccccccc}
\hline Experiment & $\begin{array}{c}\text { Thermal } \\
\text { Power } \\
\text { (GWth) }\end{array}$ & $\begin{array}{c}\text { Baselines } \\
\text { Near/Far } \\
\text { (meters) }\end{array}$ & $\begin{array}{c}\text { Overburden } \\
\text { Near/Far } \\
\text { (mwe) }\end{array}$ & $\begin{array}{c}\text { Target } \\
\text { Mass } \\
\text { (Tons) }\end{array}$ & $\begin{array}{c}\text { Start } \\
\text { Date } \\
\text { (Proj. }^{\dagger}\end{array}$ & Sensitivity* $^{*}$ \\
\hline Double Chooz & 8.6 & $410 / 1050$ & $115 / 300$ & $8.8 / 8.8$ & $2010-11$ & 0.032 \\
\hline RENO & 17.3 & $290 / 1380$ & $120 / 450$ & $16 / 16$ & 2010 & 0.02 \\
\hline Daya Bay & 17.4 & $363 / 1985$ & $255 / 910$ & $40 / 80$ & 2011 & 0.008 \\
& & $481 / 1613$ & $292 / 910$ & $40 / 80$ & & \\
\hline
\end{tabular}

* At $\Delta m_{13}^{2}=2.5 \times 10^{-3} \mathrm{eV}^{2}, 90 \%$

$\dagger$ The start dates are approximate based on current construction schedules.

** Meters water equivalent

\$ The Daya Bay site involves 2 near sites near 2 groups of reactors, so the baselines and overburdens of the 2 near sites are given separately.

\section{The Daya Bay Experiment}

The Daya Bay experiment has the best projected sensitivity for $\theta_{13}$. The Daya Bay nuclear power plant currently operates 4 reactors with a total thermal power of $11.6 \mathrm{GW}_{\text {th }}$. Two additional reactors are under construction and will begin operation in 2011, increasing the total power to $17.4 \mathrm{GW}_{\text {th }}$. The reactors are located in 2 groups with a pair at the "Daya Bay" location and the remaining at the "Ling Ao" location at about $1 \mathrm{~km}$ distance. The plant is located adjacent to mountainous terrain that facilitates construction of underground detector halls to reduce background from cosmic radiation.

The Daya Bay experiment will consist of eight detector modules, each with a target mass of 20 Tonnes. These detectors will be deployed in three experimental halls, connected by horizontal tunnels. The projected sensitivity of the Daya Bay experiment is $\sin ^{2} 2 \theta_{13}<0.008$ at $90 \%$ confidence for three years of running. The excavation of the underground tunnels and experimental halls began in Fall 2007. The full experiment should be operational in 2011.

\section{ACCELERATOR-BASED EXPERIMENTS}

A variety of long-baseline accelerator based experiments are currently underway or in preparation. If the reactor experiments can determine $\sin ^{2} 2 \theta_{13}>0.01$ then the next set of goals is to establish $C P$-violation in the PMNS matrix and determine the mass hierarchy.

The formula for $v_{e}$ appearance is given by a more complicated expression (here including matter effects to lowest order)[1]:

$$
P\left(v_{\mu} \rightarrow v_{e}\right) \simeq 4 c_{13}^{2} s_{13}^{2} s_{23}^{2} \sin ^{2} \Delta_{31}
$$




$$
\begin{aligned}
& +8 c_{13}^{2} s_{13} s_{23} c_{23} s_{12} c_{12} \sin \Delta_{31}\left[\cos \Delta_{32} \cos \delta-\sin \Delta_{32} \sin \delta\right] \sin \Delta_{21} \\
& -8 c_{13}^{2} s_{13}^{2} s_{23}^{2} s_{12}^{2} \cos \Delta_{32} \sin \Delta_{31} \sin \Delta_{21} \\
& +\quad 4 c_{13}^{2} s_{12}^{2}\left[c_{12}^{2} c_{23}^{2}+s_{12}^{2} s_{23}^{2} s_{13}^{2}-2 c_{12} c_{23} s_{12} s_{23} s_{13} \cos \delta\right] \sin ^{2} \Delta_{21} \\
& -8 c_{13}^{2} s_{13}^{2} s_{23}^{2}\left(1-2 s_{13}^{2}\right) \frac{a L}{4 E_{v}} \sin \Delta_{31}\left[\cos \Delta_{32}-\frac{\sin \Delta_{31}}{\Delta_{31}}\right] .
\end{aligned}
$$

where the notation

$$
s_{i j}=\sin \theta_{i j}, c_{i j}=\cos \theta_{i j}, \Delta_{i j}=\Delta m_{i j}^{2} L / 4 E_{v}
$$

is used and the matter effect involves the parameter

$$
a \equiv 2 \sqrt{2} G_{F} N_{e} E_{v}=1.54 \times 10^{-4} Y_{e} \rho\left(\mathrm{g} / \mathrm{cm}^{3}\right) E_{v}(\mathrm{GeV})\left(a \text { is in }\left(\mathrm{eV}^{2}\right)\right) .
$$

The probability of the conjugate process $P\left(\bar{v}_{\mu} \rightarrow \bar{v}_{e}\right)$ is obtained by the substitution $\delta \rightarrow-\delta$ and $a \rightarrow-a$.

The $v_{e}$ appearance probability clearly depends on the phase $\delta$ and the mass hierarchy [ $\left.\operatorname{sign}\left(\Delta m_{31}^{2}\right)\right]$ in addition to the mixing angle $\theta_{13}$. Observation of this process is certainly of great interest, but to extract the physics will require detailed studies to separate the effects of $C P$ violation and mass hierarchy.

\section{Near-term Experiments}

The T2K (Tokai to Kamioka) experiment is currently under construction, and is expected to begin operation in 2010 . It will utilize a neutrino beam generated by the new JPARC facility and the existing Super-Kamiokande water Cherenkov detector at a distance of $295 \mathrm{~km}$. Running will begin with low-power, $0.1 \mathrm{MW}$, with the ultimate goal of reaching $0.75 \mathrm{MW}$. With 5 years running at $0.75 \mathrm{MW}$ the sensitivity to $\sin ^{2} 2 \theta_{13}$ will approach 0.01 .

The NOvA experiment [19] has just begun construction and is expected to begin operation in 2013. The existing main injector neutrino beam (NuMI) at Fermilab will be employed, but in an off-axis (14 mr) geometry to the new Ash River far detector site in Minnesota $(811 \mathrm{~km})$. The beam power will be upgraded to $0.7 \mathrm{MW}$. The detector is a $14 \mathrm{kT}$ array of $4 \mathrm{~cm} \times 6 \mathrm{~cm} \times 15 \mathrm{~m}$ liquid scintillator modules, enabling excellent electron identification. The run plan is for 3 years of $v_{\mu}$ and 3 years of $\bar{v}_{\mu}$ with a sensitivity to $\sin ^{2} 2 \theta_{13} \sim 0.01$ at $90 \%$ CL. If $\sin ^{2} 2 \theta_{13}$ is significantly larger than 0.01 , NOvA could have sensitivity to the mass hierarchy as well as the $C P$ violating phase $\delta$.

\section{Future Proposed Project at DUSEL}

During the last few years a new future project to study long baseline neutrino oscillations has been developed in the United States. The basis for this concept was a joint Fermilab/Brookhaven study [20] that considered various options with detailed simulations. In 2008, the Particle Physics Prioritization Panel (P5) endorsed the plan to site 
a large detector at the proposed DUSEL (Deep Underground Science and Engineering Laboratory) in Lead, South Dakota and construct a new neutrino beam from Fermilab directed at this location [21].

The primary goal of this experiment is to study the oscillation channels $v_{\mu} \rightarrow v_{e}$ and $\bar{v}_{\mu} \rightarrow \bar{v}_{e}$ (see Eq. 7) and determine the three quantities $\theta_{13}, \delta$ (CP violation) and the sign of $\Delta m_{13}^{2}$ (mass hierarchy). For the longer baseline of $1300 \mathrm{~km}$, there are oscillation maxima at $\Delta_{32} \simeq \frac{\pi}{2}, \frac{3 \pi}{2}$, corresponding to $E_{v} \simeq 2.5,0.8 \mathrm{GeV}$. In addition, the matter effects are quite significant at this larger distance. The experiment strategy is to produce a wide band beam that will cover the energy region of these two oscillation maxima. At this larger baseline, and with the additional capability to study $\bar{v}$, the appearance signals contain enough information to determine all three oscillation parameters.

The detector will be located deep underground at DUSEL, and a very large detector mass must be employed to yield sufficient statistical precision. In addition, the detector must provide particle identification to distinguish the electron appearance events from background neutral current $\pi^{0}$ events. Two detector technologies are under consideration for this project: water Cherenkov and liquid argon Time Projection Chamber (TPC).

It should be mentioned that such large underground detectors as described here would enable very interesting additional science goals. These additional capabilities include extending the reach for detection of nucleon decay, galactic and extra-galactic supernova neutrino detection, high-precision solar $v$ studies, and detailed studies of atmospheric neutrinos.

\section{CONCLUSIONS}

Clearly the last decade has produced remarkable progress in our understanding of neutrino oscillations and masses. The mass splittings are now well-determined and we have good quantitative knowledge of two of the three mixing angles: $\theta_{12}$ and $\theta_{23}$. The last unknown mixing angle $\theta_{13}$ has eluded experimental discovery thus far, with the $\mathrm{CHOOZ}$ experiment providing only an upper limit. The presence of $C P$ violation (through the phase $\delta$, see Eq. 3) in the neutrino mixing matrix requires that $\theta_{13} \neq 0$. Such $C P$ violation would lend support to the leptogenesis scenario [22] for generating the matter-antimatter asymmetry in the universe, and so its experimental study is of great interest. Determination of the value of $\theta_{13}$ is essential in the planning and design of future neutrino oscillation experiments to determine $\delta$ as well as the mass hierarchy.

Establishing that $\theta_{13} \neq 0$ is now a major priority for the experimental program. The three reactor experiments (RENO, Double CHOOZ, and Daya Bay) along with the new long baseline experiment $\mathrm{T} 2 \mathrm{~K}$ are preparing to address $\theta_{13}$ with higher precision, down to the level of $\sin ^{2} 2 \theta_{13}<0.01$. This represents an order of magnitude improvement in sensitivity over present experiments.

If it is indeed found that $\sin ^{2} 2 \theta_{13}>0.01$, then future long baseline experiments such as NOvA and the proposed Fermilab to DUSEL experiment will have good sensitivity to the $C P$-violating phase $\delta$ and the mass hierarchy. However, if $\theta_{13}$ is much smaller then the future determination of $\theta_{13}$ will be much more difficult. The Fermilab to DUSEL experiment could have sensitivity down to $\sin ^{2} 2 \theta_{13} \sim 2-3 \times 10^{-3}$, but measuring $\delta$ and the mass hierarchy would be much more difficult with this experiment if $\theta_{13}$ is 
that small. Thus it seems that if $\sin ^{2} 2 \theta_{13} \ll 0.01$ future experimental efforts to study $C P$ violation and the mass hierarchy will likely require new facilities with even greater capability. Present concepts include "beta beams" from radioactive decay of nuclei in storage rings [23] or "neutrino factories" that produce neutrinos from the decay of stored muons [24]. These concepts are presently undergoing research and development to assess the ultimate feasibility for future construction.

\section{ACKNOWLEDGMENTS}

It is a pleasure to acknowledge fruitful discussions with Petr Vogel. This work was supported in part by NSF grant PHY-0855538.

\section{REFERENCES}

1. R. D. McKeown and P. Vogel, Phys. Rep. 394315 (2004).

2. W. Pauli, letter to a physicist's gathering at Tubingen, December 4, 1930. Reprinted in Wolfgang Pauli, Collected Scientific Papers, ed. R. Kronig and V. Weisskopf, Vol. 2, p.1313 (Interscience: New York, 1964).

3. C. L. Cowan, Jr., F. Reines, F. B. Harrison, H. W. Kruse, and A. D. McGuire, Science 124, 103 (1956); F. Reines and C. L. Cowan, Phys. Rev. 92, 830 (1953).

4. B. Pontecorvo, Sov. Phys. JETP 6, 429 (1958); B. Pontecorvo, Sov. Phys. JETP 33, 549 (1967).

5. R. Davis Jr., D. S. Harmer, and K. C. Hoffman, Phys. Rev. Lett. 20, 1205 (1968).

6. V. Rubin, W. K. Ford, Jr., Astrophys. J. 159, 379 (1970); V. Rubin, N. Thonnard, and W. K. Ford, Jr., Astrophys. J. 238, 471, (1980).

7. Y. Fukuda, et al., Phys. Rev. Lett. 81, 1562 (1998).

8. Z. Maki, M. Nakagawa and S. Sakata, Prog. Theor. Phys. 28, 870 (1962).

9. Y. Ashie, et al., Phys. Rev. D71, 112005 (2005).

10. J. M. Paley, et al.,, arXiv:0901.2131.

11. C. Bemporad, G. Gratta and P. Vogel, Rev. Mod. Phys. 74, 297 (2002).

12. P. Vogel and J. F. Beacom, Phys. Rev. D60, 053003 (1999); A. Kurylov, M.J. Ramsey-Musolf, and P. Vogel, Phys.Rev. C67 035502 (2003).

13. M. Appolonio et al., Eur. Phys. J. C27, 331 (2003)

14. F. Boehm, et al., Phys. Rev. D64, 112001 (2001).

15. S. Abe, et al., Phys. Rev. Lett. 100, 221803 (2008).

16. F. Ardellier et al., Double Chooz: A Search for the Neutrino Mixing Angle $\theta_{13}$, arXiv:hep-ex/0606025 (2006).

17. Daya Bay Collaboration, A Precision Measurement of the Neutrino Mixing Angle $\theta_{13}$ using Reactor Antineutrinos at Daya Bay, hep-ex/0701029 (2007).

18. K.K.Joo for the RENO collaboration, Nucl. Phys. B168, 125 (2007).

19. D. S. Ayers, et al., NOvA Technical Design Report, FERMILAB-DESIGN-2007-01, http://wwwnova.fnal.gov/nova_cd2_review/tdr_oct_23/tdr.htm (2007).

20. Fermilab-0801-AD-E, BNL-77973-2007-IR, (May 9, 2007) http://nwg.phy.bnl.gov/ diwan/nwg/fnalbnl/report.pdf .

21. Particle Physics Project Prioritization Panel (P5) US Particle Physics: Scientific Opportunities. A Strategic Plan for the Next Ten Years (June 2, 2008), http://www.science.doe.gov/hep/files/pdfs/P5_Report06022008.pdf .

22. M. Fukugita and T. Yanagida, Phys. Lett. B174, 45 (1986).

23. P. Zucchelli, Phys. Lett. B532, 166 (2002); M. Mezzetto, hep-ex/0302007.

24. J. J. Gomez-Cadenas and D. A. Harris, Ann. Rev. Nucl. Part. Sci. 52, 253 (2002); P. Huber, M. Lindner and W. Winter, Nucl. Phys. B645, 3 (2002); V. Barger et al., Phys. Rev. D62, 073002 (2000); S. Geer, J. Phys. G. 29, 1485 (2003). 